\title{
INCORPORATING INCIDENCE ANGLE VARIATION INTO SAR IMAGE SEGMENTATION
}

\author{
Anthony P. Doulgeris and Anca Cristea \\ UiT - The Arctic University of Norway, Tromsø, Norway.
}

\begin{abstract}
We present a new approach for incorporating incidence angle derived synthetic aperture radar (SAR) brightness variation directly into SAR image analysis. This approach is unique in that the incidence angle dependency is modeled explicitly into the probability density function rather than an image-wide pre-processing 'correction'. It can then be used for supervised and unsupervised image analysis, and is notably able to account for a different dependency rate for each class. This has potential benefits for wide-swath SAR imagery over flat areas and ocean, wide angled airborne and UAV based SAR data, connecting narrow-beam SAR images at different acquisition angles, as well as land-based analysis with local topographic terrain angles. An initial example demonstrates unsupervised image segmentation applied to sea ice mapping for meteorological services and climate science, and is compared to the same algorithm without the incidence angle modeling.
\end{abstract}

Index Terms - Synthetic Aperture Radar, Incidence Angle Correction, Wide-swath imagery, Terrain Correction, Sentinel-1

\section{INTRODUCTION}

Wide-swath SAR images, such as from Sentinel-1, cover hundreds of kilometers, but this wide coverage, and side-looking SAR geometry, means that the imaged pixels are viewed over a large range of incidence angles. The incidence angle (IA) affects the microwave interaction with the surface properties, produces varying backscatter brightness from near range to far range and thus confuses image interpretation. The brightness variations are usually much greater than the class-toclass differences and will significantly affect image classification. This work shall initially explore wide-swath Sentinel-1 imagery for Arctic sea ice monitoring, where the brightness variation of wide swath SAR imaging has restricted its use for operational services.

Our research group, at UiT - The Arctic University of Norway, has developed advanced and automatic algorithms for analyzing narrow-swath quad-polarization SAR images

This work was funded by the Akademiaavtale between Statoil and the Arctic University of Norway, and by CIRFA partners and the Research Council of Norway (grant number 237906). over sea ice, where the IA variation is minimal and can generally be ignored $[1,2,3,4]$. However, two distinct problems were encountered when applying these same algorithms to wide-swath SAR imagery, in particular from Sentinel-1. The first relates to the IA dependency already noted, and produced too many classes, essentially over-segmenting into bands or zones in the range direction. The second relates to the variable noise-floor patterns in the range direction from stitching the different acquisition strips into the one image, which was reduced by using the thermal noise removal method from the ESA's SNAP software. Both problems and the ice type dependencies are well described in [5].

This IA effect has strong foundations in the physical interaction of the electro-magnetic microwave signal with the surface properties such as roughness, and can be described with Lambert's cosine law, Minnaert's law, or approximated with an exponential relation or log-linear model [5]. Many research papers for ocean surface modeling, particularly sea ice monitoring, have published the observed decay rates for this effect, and have demonstrated that it depends on the particular surface properties, i.e., the terrain type, most likely due to differing surface roughness. The traditional, and practical, approach is to apply a mean IA rate to the entire intensity image in a pre-processing stage, and is known as incidence angle correction or compensation. This means that a single imagewide rate is applied to all pixels and does not account for the terrain-type dependent relations, but is all that can be considered before the terrain type is known. However, in a circular fashion, the IA variation will affect the ability of classification algorithms to determine the terrain type, because it broadens the class probability distributions making image-wide classification less distinct. The single image-wide IA correction will at best be correct for only one terrain class and may over or under-correct other classes.

Our solution to account for this systematic IA dependency is to incorporate the IA variation directly into our statistical probability models that are used for supervised classification and unsupervised segmentation (clustering). This approach is unique and the first to automatically and simultaneously distinguish the terrain type and determine the different IA rates for each class.

The first simplified prototype has shown promising results for sea ice and ocean surface SAR image segmentation of wide-swath Sentinel-1 imagery (publication in prep.), and 
will be demonstrated here.

Our example focuses on using Sentinel-1 wide-swath images over sea ice and ocean, since they are freely available, routinely acquired for operational monitoring, and we have good cooperation with the Norwegian Meteorological office and the Norwegian Polar Institute for this topic. We will demonstrate unsupervised segmentation, as it is more difficult than supervised classification, but additional knowledge is needed to label the classes. Comparison will be made between our previous non-incident-angle algorithm to highlight the clear effect of incorporating the IA into the modeling.

The method is generic and should also work without modification on RADARSAT- 2 or any other wide-swath satellite data, and equivalently on wide-angled airborne SAR systems. We foresee valuable extensions to digital elevation model (DEM) based local terrain angle analyses, and supervised classification across multiple images acquired at different incidence angles.

\section{THEORY}

The brightness decay observed in wide-swath SAR images from near-range to far-range is a physical phenomenon relating to illumination and scattering, and probably has several contributing factors. Ideal diffuse reflections may follow Lambert's law of illumination [6], which describes how the radiant intensity from an ideal diffuse emitter decreases from an emission at $\theta=0^{\circ}$ to an emission at $\theta=90^{\circ}$ proportionally to the cosine of the angle $\theta$. Furthermore, the observed radiance varies as a function of the observed area according to the same law. For microwave remote sensing, the single antenna acts both as the emitter and the receiver and therefore the received intensity is proportional to the squared cosine of the incidence angle $\theta_{i}$ [7].

Lambert's law was extended by Minnaert [8] to the case of non-ideal diffuse emitters often observed in the real world:

$$
I_{i}=I_{0} \cos ^{2 k}\left(\theta_{i}\right)
$$

where $I_{0}$ is the radiance along the normal direction $\left(\theta_{0}=0^{\circ}\right)$, and $k$ is a roughness-dependent exponent called the Minnaert constant to represent the less than ideally diffuse scattering. Minnaert's law gives a simple mechanism to have different rates for different classes and was used for conducting classbased IA compensation [9] [10], albeit manually applied, as opposed to an ideal Lambert law-based global compensation over the entire image [11].

Experimental observations indicated that a simple exponential approximation was sufficient in many practical cases[5] [12]:

$$
I_{i}=I_{0} e^{-\theta_{i} / \theta_{0}}
$$

where $\theta_{0}$ represents the decay rate that could be different per class like Minnaert's $k$ parameter. By using the same terms as in [12], the above relation can be linearized so that the logintensity $[\mathrm{dB}]$ decays linearly as a function of the IA:

$$
I_{i}[d B]=a+b \theta_{i}
$$

where $a$ relates to $I_{0}$ and $b$ (although negative) relates to Minnaert's rate $k$. These coefficients depend on the surface scattering properties and are essentially extending the definition of the associated class.

\section{METHODS}

We have previously developed automatic segmentation methods based on the statistics of SAR polarimetry data, from a simple mixture of Gaussian features model [4] to variations using more complicated textured models [2]. We now present the first simplified version incorporating the incidence-angle dependency directly into the statistical model.

For one specific class, we consider its log-intensity along constant-incidence angle azimuth lines to follow a Gaussian distribution with a mean value expressed as a linear function of the given IA (eq. 3). For two channel data, for example Sentinel-1 HH and HV images, we allow different decay parameters for each channel, as well as each class, and thus $a$ and $b$ are multivariate. The resulting mixture distribution of $M$ classes for all image pixels, with class priors $\pi_{m}$, and class covariance matrix $\Sigma_{m}$, is thus described by a set of parameters, $\left(\pi_{m}, a_{m}, b_{m}, \Sigma_{m}\right)$ for each class $m$, and modeled by the $d$-dimensional distribution:

$p(x, \theta)=\sum_{m=1}^{M} \frac{\pi_{m}}{(2 \pi)^{\frac{d}{2}} \Sigma^{\frac{1}{2}}} e^{-\frac{1}{2}\left(x-\left(a_{m}+b_{m} \theta\right)\right)^{T} \Sigma^{-1}\left(x-\left(a_{m}+b_{m} \theta\right)\right)}$

The mixture is separated using the Expectation-Maximization algorithm, which (in this simple case) has closed-form update expressions, i.e. :

$$
\begin{gathered}
a_{m}=\frac{\sum_{i=1}^{n} z_{i m} x_{i}-b_{m} \sum_{i=1}^{n} z_{i m} \theta_{i}}{\sum_{i=1}^{n} z_{i m}} \\
b_{m}=\frac{\sum_{i=1}^{n} z_{i m} \theta_{i} x_{i}-a_{m} \sum_{i=1}^{n} z_{i m} \theta_{i}}{\sum_{i=1}^{n} z_{i m} \theta_{i}^{2}} \\
\Sigma_{m}=\frac{\sum_{i=1}^{n} z_{i m}\left(x_{i}-\left(a_{m}+b_{m} \theta_{i}\right)\right)\left(x_{i}-\left(a_{m}+b_{m} \theta_{i}\right)\right)^{T}}{\sum_{i=1}^{n} z_{i m}}
\end{gathered}
$$

where $z_{i m}$ denotes the expected [posterior] probability that the pixel $i$ belongs to the class $m$.

\section{RESULTS}

Our example is an Extra-Wide swath (EW) Sentinel-1 image acquired over the Fram Strait during the freeze-up season of 2015 (Fig. 1). The image was selected because of the clearly visible classes extending along the range direction. Ocean 
water along the top quarter of the image, and sea ice elsewhere, as seen in the greyscale intensity images of Fig. 1 (a) and (b). This provides a clear demonstration of the algorithm's ability to connect near-range and far-range regions that belong to the same class.

The mixture-of-Gaussians based segmentation algorithm was applied directly to multi-looked $\mathrm{HH}$ and HV intensities [in decibels], and automatically converged when the model shows a good fit to all classes. For simpler interpretation, and in light of the Gaussian approximation, we multi-looked and additional $5 \times 5$ (over the 18-look product) and sub-sampled the image (to 20000 pixels) to reduce the number of distinguishable clusters presented. Fig. 1 (c) shows the segmentation results obtained without incidence-angle correction, and Fig. 1 (d) shows the proposed incidence-angle modeling results. The effect of the incidence-angle correcting segmentation over the non-correcting one is clear, as the division of the classes into multiple bands according to the decaying intensities along the range dimension is no longer visible.

Fig. 1 (e) shows the decay rates of the HH Intensity [dB] for all classes in (d). The high decay rates of the water classes (red and yellow) are clearly distinguishable from the lower rates corresponding to the compact sea ice class (green) and potentially frozen leads class (blue).

\section{CONCLUSION}

We introduce an automatic segmentation method for wideswath PolSAR images that incorporates class-based incidence angle variation, and demonstrate that the algorithm is functional and delivers good results which hold a physical meaning, albeit for a simplified case. Improvements may be achieved using a more flexible incidence-angle relation, and by changing the statistical model to a more appropriate one that captures the details of the heavy tails, such as the Gamma or a compound distribution containing a texture term. It could also prove worthwhile to test whether the polarimetric features, previously used for segmentation, have decay rates follow the same law as the intensities.

This modeling approach is quite generic and should work with all wide incidence-angle ranged data, for supervised classification across different acquisition ranges, as well as for DEM based terrain angle compensation.

\section{REFERENCES}

[1] A. M. Johansson, J. A. King, A. P. Doulgeris, S. Gerland, S. Singha, G. Spreen, and T. Busche, "Combined observations of Arctic sea ice with near-coincident colocated X-band, C-band, and L-band SAR satellite remote sensing and helicopter-borne measurements," Journal of Geophysical Research: Oceans, vol. 122, no. 1: 669691, 2017.
[2] Anthony P. Doulgeris, “An Automatic U-Distribution and Markov Random Field Segmentation Algorithm for PolSAR Images," IEEE Transactions on Geoscience and Remote Sensing, vol. 53, no. 4: 1819-1827, 2015.

[3] M. A N Moen, S. N. Anfinsen, A. P. Doulgeris, A. H H Renner, and S. Gerland, "Assessing polarimetric SAR sea-ice classifications using consecutive day images," Annals of Glaciology, vol. 56, no. 69: 285-294, 2015.

[4] Anthony P. Doulgeris, "A Simple and Extendable Segmentation Method for Multi-Polarisation SAR Scenes," in The 6th International Workshop on Science and Applications of SAR Polarimetry and Polarimetric Interferometry (POLinSAR 2013), Frascati, Italy, January 28 February 1 2013, 2013.

[5] Marko Makynen and Juha Karvonen, "Incidence Angle Dependence of First-Year Sea Ice Backscattering Coefficient in Sentinel-1 SAR Imagery Over the Kara Sea," IEEE Transactions on Geoscience and Remote Sensing, vol. 55, no. 11: 6170-6181, 2017.

[6] J.H. Lambert, Photometria, sive de Mensura et Gradibus Luminis, Colorum et Umbrae, Augsburg, 1760.

[7] F. T. Ulaby, R. K. Moore, and A. K. Fung, Microwave Remote Sensing: Active and Passive. Volume II-Radar Remote Sensing and Surface Scattering and Emission Theory, Norwood, MA: Artech House, 1982.

[8] M. Minnaert, "The Reciprocity Principle in Lunar Photometry," The Astrophysical Journal, vol. 93: 403, 1941.

[9] Juan Pablo Ardila, Valentyn Tolpekin, and Wietske Bijker, "Angular Backscatter Variation in L-band ALOS ScanSAR Images of Tropical Forest Areas," IEEE Geoscience and Remote Sensing Letters, vol. 7, no. 4: 821825, 2010.

[10] Wenhui Lang, Pan Zhang, Jie Wu, Yang Shen, and Xuezhi Yang, "Incidence Angle Correction of SAR Sea Ice Data Based on Locally Linear Mapping," IEEE Transactions on Geoscience and Remote Sensing, vol. 54, no. 6: 3188-3199, 2016.

[11] Konstantinos Topouzelis and Suman Singha, "Incidence angle Normalization of Wide Swath SAR Data for Oceanographic Applications," Open Geosciences, vol. 8, no. 1: 450-464, 2016.

[12] Zihang Zhao, Wenhui Lang, Anthony Paul Doulgeris, and Lu Chen, "Improved Llm Methods Using Linear Regression," in IEEE International Geoscience and Remote Sensing Symposium proceedings, 2017: 53505353. 




(a) HH Intensity $[\mathrm{dB}]$

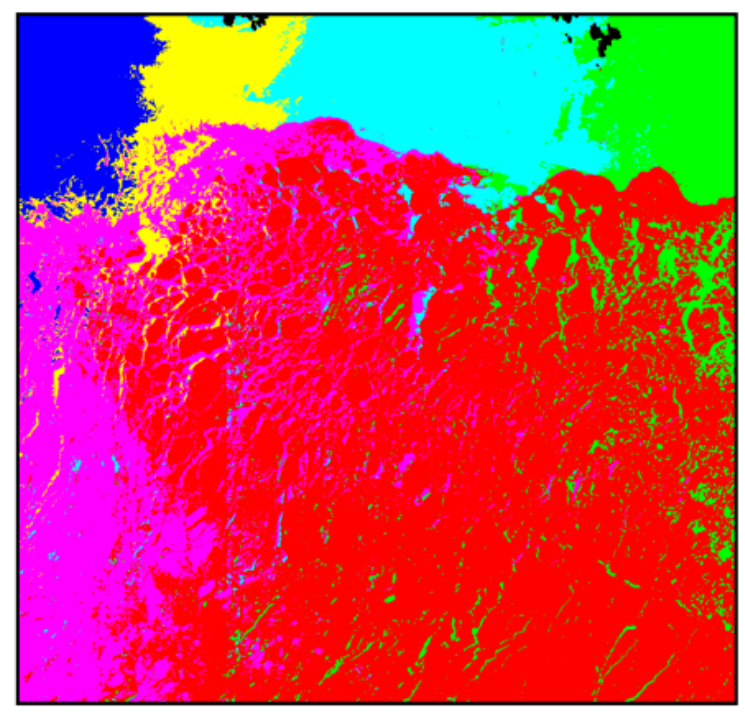

(c) Segmentation without IA

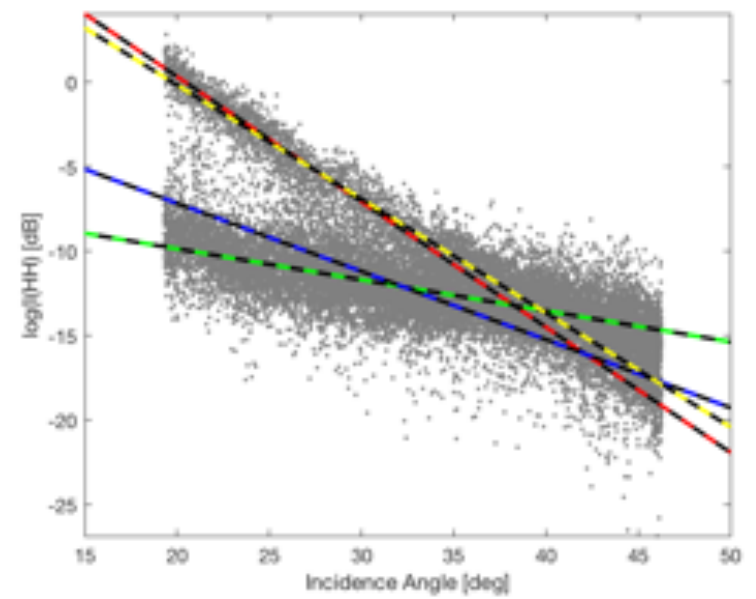

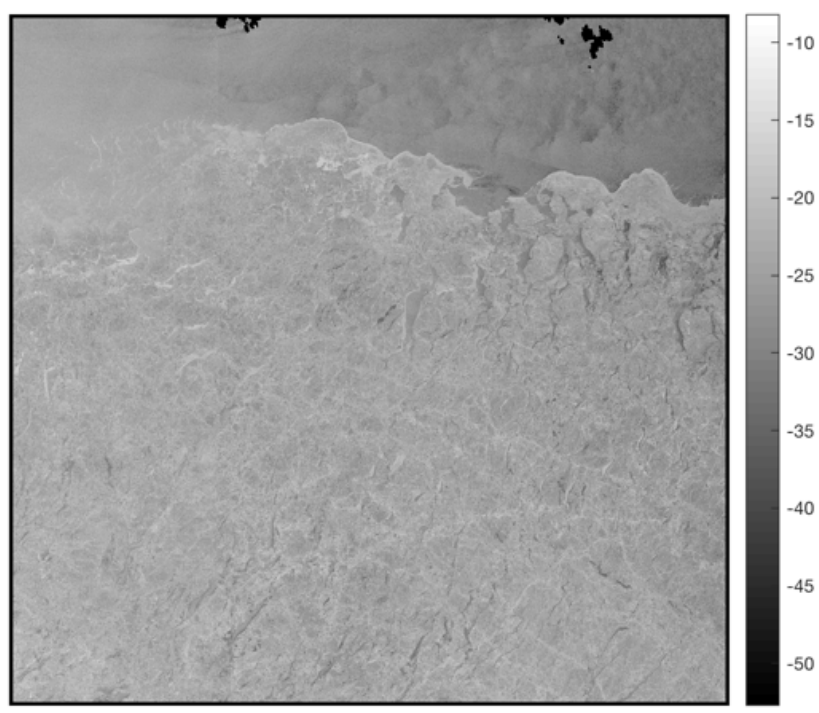

(b) HV Intensity [dB]

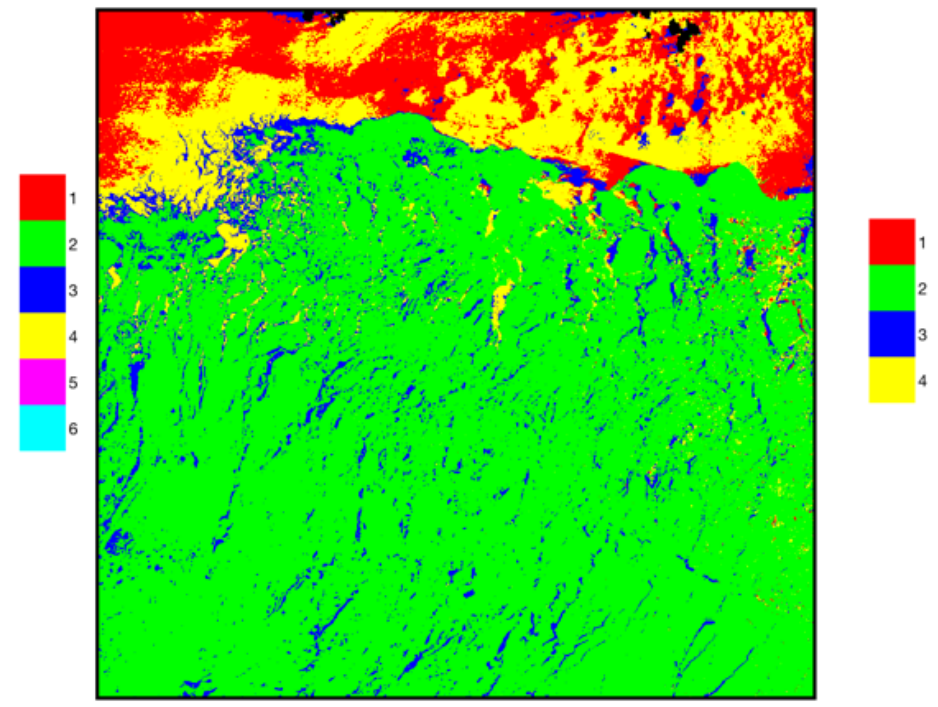

(d) (new) Segmentation with IA

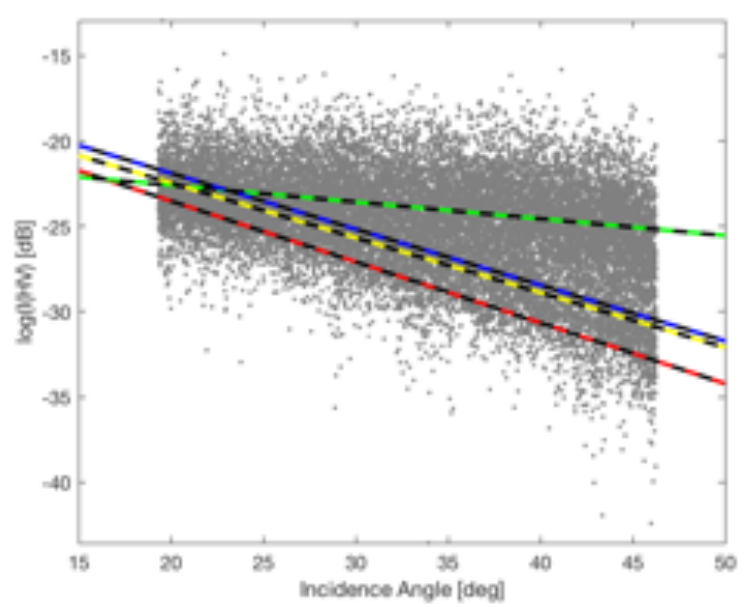

(e) Class dependent IA rates

Fig. 1. Example of Sea Ice (lower part of image) and Ocean (top part of image) segmentation. Note how the banding due to incidence angle variation is automatically removed with the new approach, and the log-linear class dependencies from (d) are shown in (e). 